\title{
Coronal Three-Dimensional Magnetic Resonance Imaging for Improving Diagnostic Accuracy for Posterior Ligamentous Complex Disruption In a Goat Spine Injury Model
}

\author{
Xuee Zhu, MD, Jichen Wang, MD, PhD, Dan Zhou, MD, Chong Feng, MD, Zhiwen Dong, MD, \\ Hanxiao Yu, MD \\ All authors: Department of Radiology, BenQ Medical Center, The Affiliated BenQ Hospital of Nanjing Medical University, Nanjing, China
}

\begin{abstract}
Objective: The purpose of this study was to investigate whether three-dimensional (3D) magnetic resonance imaging could improve diagnostic accuracy for suspected posterior ligamentous complex (PLC) disruption.

Materials and Methods: We used 20 freshly harvested goat spine samples with 60 segments and intact surrounding soft tissue. The animals were aged 1-1.5 years and consisted of 8 males and 12 females, which were sexually mature but had not reached adult weights. We created a paraspinal contusion model by percutaneously injecting $10 \mathrm{~mL}$ saline into each side of the interspinous ligament (ISL). All segments underwent T2-weighted sagittal and coronal short inversion time inversion recovery (STIR) scans as well as coronal and sagittal 3D proton density-weighted spectrally selective inversion recovery (3D-PDW-SPIR) scans acquired at 1.5T. Following scanning, some ISLs were cut and then the segments were rescanned using the same magnetic resonance (MR) techniques. Two radiologists independently assessed the MR images, and the reliability of ISL tear interpretation was assessed using the kappa coefficient. The chi-square test was used to compare the diagnostic accuracy of images obtained using the different MR techniques.

Results: The interobserver reliability for detecting ISL disruption was high for all imaging techniques (0.776-0.949). The sensitivity, specificity, and diagnostic accuracy of the coronal 3D-PDW-SPIR technique for detecting ISL tears were 100, 96.9 , and $97.9 \%$, respectively, which were significantly higher than those of the sagittal STIR $(p=0.000)$, coronal STIR ( $p$ $=0.000)$, and sagittal 3D-PDW-SPIR $(p=0.001)$ techniques.

Conclusion: Compared to other MR methods, coronal 3D-PDW-SPIR provides a more accurate diagnosis of ISL disruption. Adding coronal 3D-PDW-SPIR to a routine MR protocol may help to identify PLC disruptions in cases with nearby contusion. Keywords: Spine; Animal model; Trauma; MRI
\end{abstract}

\section{INTRODUCTION}

The posterior ligamentous complex (PLC), which is composed of the supraspinous ligament (SSL), interspinous ligament (ISL), ligamentum flavum (LF), and facet joint capsule $(\mathrm{FC})$, protects the spine from excessive translation, rotation, flexion, and distraction. PLC injuries are major causes of spinal instability (1-4). According to the recently established Thoracolumbar Injury Classification and Severity Score (TLICS), PLC integrity is an important consideration for surgical candidacy and may influence the surgical approach used $(5,6)$. Therefore, in clinical settings, it is

Received September 9, 2018; accepted after revision December 23, 2018.

This study was supported by the Medical Science and Technology Development Project of Nanjing, China (grant no. YKK15214).

Corresponding author: Jichen Wang, MD, PhD, Department of Radiology, BenQ Medical Center, The Affiliated BenQ Hospital of Nanjing Medical University, 71 Hexi Ave, Jianye District, Nanjing 210019, China.

- Tel: (86) 13951804606• Fax: (8625) 52238800-3377• E-mail: fskwjc@126.com

This is an 0pen Access article distributed under the terms of the Creative Commons Attribution Non-Commercial License (https:// creativecommons.org/licenses/by-nc/4.0) which permits unrestricted non-commercial use, distribution, and reproduction in any medium, provided the original work is properly cited. 
necessary to accurately evaluate PLC injuries before surgery.

The methods used for clinically assessing the PLC include physical examination, ultrasonography (US), plain radiography, computed tomography (CT), and magnetic resonance imaging (MRI). Of these methods, palpation is the most unreliable and inaccurate (7). US is operatordependent. Although plain radiography and $\mathrm{CT}$ allow visualization of osseous anatomy, these methods provide only indirect evidence of ligamentous injuries. MRI can directly detect soft tissue injuries and is the preferred means of diagnosing ligamentous injuries (7-14). However, some studies have reported that MRI was highly sensitive but had a poor positive predictive value, suggesting an unacceptable false positive rate $(2,14,15)$. Moreover, some authors have questioned the role of MRI in assessing PLC integrity $(2,15)$.

Normally, ligaments show low signal intensity on all MRI sequences. On T2-weighted sequences, the loss of ligamentous continuity or presence of fluid crossing the location of the ligament are specific signs of ligamentous tears. In most previous studies, these exact criteria were used for diagnosing SSL and LF disruption $(2,5$, $8,9,12,16-23)$. In these studies, ISL injuries were diagnosed by observation of increased signal intensity (i.e., hyperintensity), and FC injuries were indicated by the presence of articular liquid or diastasis of the facet joint. Hyperintensity on fluid-sensitive sequences suggests edema, hemorrhage, or inflammation. These findings are associated with ligamentous disruption or bone fractures but do not necessarily indicate the presence of a ligamentous tear. Using hyperintensity as a standard sign for diagnosing ligamentous disruption inevitably lowers diagnostic specificity.

Three-dimensional (3D) MRI allows for thinner slice imaging with no interslice gaps and enables multiplanar reconstructed images to be viewed in any plane. Therefore, the purpose of this study was to investigate whether 3D MRI could improve diagnostic accuracy for suspected PLC disruption.

\section{MATERIALS AND METHODS}

This study was approved by the Animal Care Committee at our institution. We used 20 freshly harvested goat spine samples with 60 segments (i.e., 4 T12-L1, 8 L1-L7, 8 L7S1) and intact surrounding soft tissue. The animals were aged 1-1.5 years and consisted of 8 males and 12 females, which were sexually mature but had not reached adult weights $(22-30 \mathrm{~kg})$.

Initially, all of the intact segments underwent sagittal followed by coronal T2-weighted short inversion time inversion recovery (STIR) scans (repetition time, 2500 ms; echo time, $65 \mathrm{~ms}$; flip angle, $90^{\circ}$; echo train length, 4; number of excitations, 2; slice thickness, $3.5 \mathrm{~mm}$; intersection gap, $3.5 \mathrm{~mm}$; matrix, $180 \times 204$; and field of view, $180 \times 296 \mathrm{~mm}$ ). Following these scans, 3D proton density-weighted spectrally selective inversion recovery (3D-PDW-SPIR) scanning was performed in the coronal plane, and later images were reconstructed in the sagittal plane. The 3D-PDW-SPIR parameters were as follows: repetition time, $850 \mathrm{~ms}$; echo time, $35 \mathrm{~ms}$; flip angle, $90^{\circ}$; echo train length, 4; number of excitations, 3; slice thickness, $1.6 \mathrm{~mm}$; matrix, $200 \times 190$; and field of view, 160 $\times 160 \mathrm{~mm}$. All imaging was performed with a spine surface coil. A rice pad was wrapped around each specimen to improve the fat suppression effect. All images were acquired using a 1.5T MRI scanner (Intera; Philips Healthcare, Best, The Netherlands).

To mimic a paraspinal soft tissue contusion, we percutaneously injected $10 \mathrm{~mL}$ saline into each side of the ISL. Injection around the FC was avoided since the FC is difficult to distinguish on STIR and 3D-PDW-SPIR images. Following saline injection, the segments underwent repeated MRI using the aforementioned protocol.

Following MRI, the interspinous space of every specimen was cut under fluoroscopic control using a metal crochet hook (Fig. 1A, B) and the segments were re-scanned using the aforementioned protocol. After acquiring the final MRI scans, each specimen underwent meticulous anatomical dissection by an operator who was blind to the cutting procedure and magnetic resonance (MR) images. The ISL status of each specimen was confirmed by direct visualization (Fig. 1C).

Using two scales for intact and disrupted structures, two senior musculoskeletal radiologists (i.e., one spine radiology specialist with 10 years of experience and one radiologist with 5 years of experience) independently and blindly evaluated the MR images to assess ISL integrity on sagittal and coronal STIR as well as coronal and sagittal 3D-PDW-SPIR images. ISL disruptions were diagnosed by the following MRI findings: 1 . Discontinuity of the black stripe (i.e., low signal intensity) representing the ISL or fluid crossing the location of the ISL on coronal images (Fig. 2A, B) and 2. Linear hypersignal intensity in the 


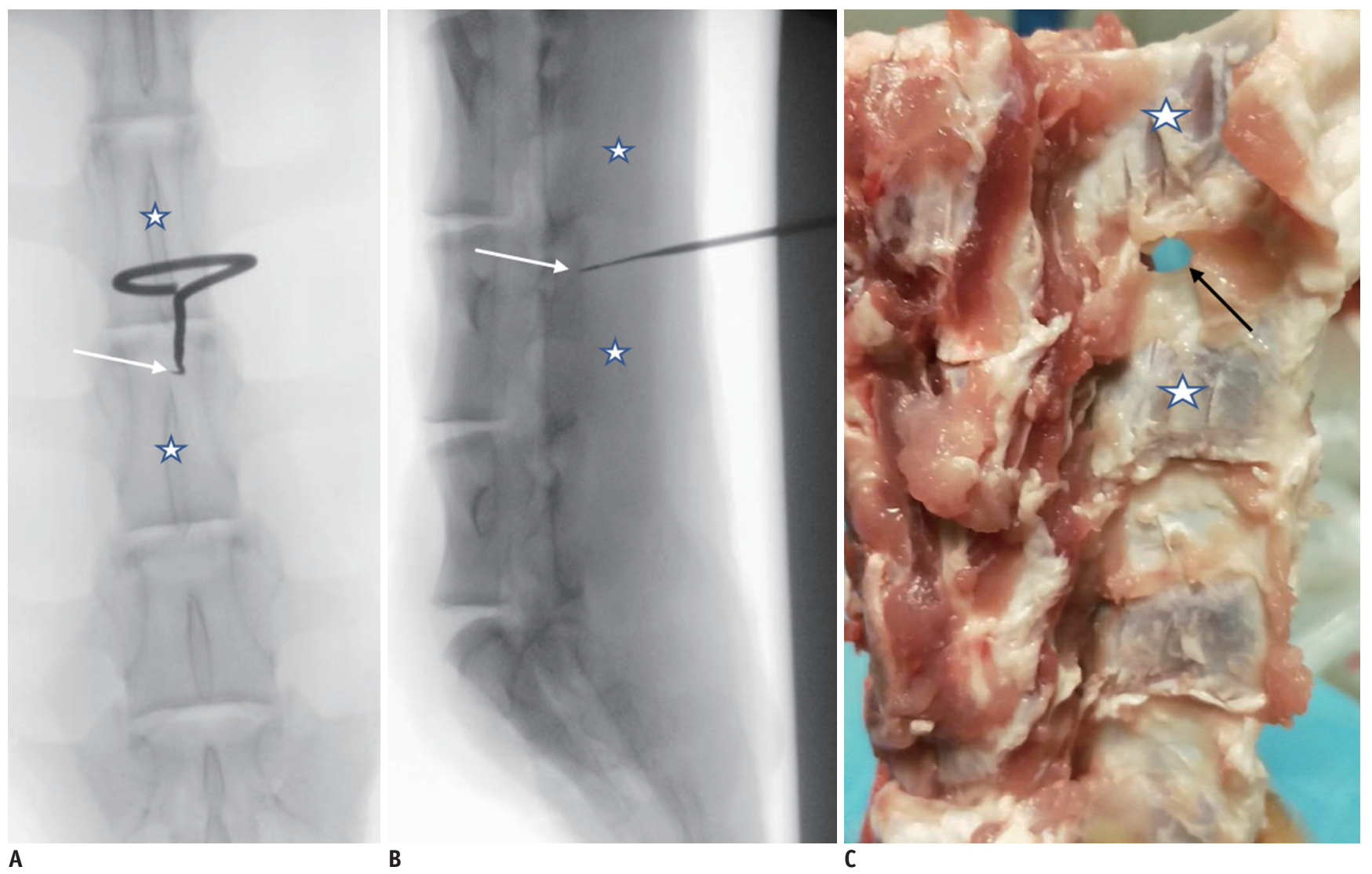

\section{Fig. 1. Percutaneous ISL resection.}

Posteroanterior (A) and lateral (B) fluoroscopic views show metal crochet hook tip (white arrows) placed in anterior portion of interspinous space, which was identified between two adjacent spinous processes (stars). C. ISL was successfully cut out (black arrow) as shown in following dissection. 'Stars' in image $\mathbf{C}$ indicate spinous processes. ISL = interspinous ligament

interspinous space on sagittal images (Fig. 2C, E). However, on sagittal images, areas of diffuse high signal intensity in the interspinous space only indicated adjacent soft tissue contusion (Fig. 2D, F).

The MRI interpretations were compared with later dissection findings to determine the diagnostic accuracy, sensitivity, and specificity. The chi-square test was used to compare the diagnostic accuracy of images obtained using sagittal STIR, coronal STIR, coronal 3D-PDW-SPIR, and sagittal 3D-PDW-SPIR. A $p$ value $<0.05$ was considered significant. The reliability of the two radiologists in interpreting ISL tears was assessed using the kappa coefficient $(\kappa)$. The $\kappa$ values were defined as slight ( $0-$ $0.20)$, fair $(0.21-0.40)$, moderate $(0.41-0.60)$, substantial (0.61-0.80), and almost perfect (0.81-1.00). Statistical analyses were performed using STATA/SE software (version 14; StataCorp, College Station, TX, USA).

\section{RESULTS}

\section{Delineation of Normal ISL and FC on MR Images}

The FC was not clearly visualized on STIR or 3D-PDWSPIR images due to its small size. The ISL presented as areas of diffuse homogeneous low signal intensity in the interspinous space on sagittal STIR and 3D-PDW-SPIR images. Moreover, the outline of the ISL was unclear on these images. On coronal STIR and 3D-PDW-SPIR images, the ISL appeared as a thin black stripe with a well-defined border (Fig. 3).

Interestingly, on two-dimensional (2D) and 3D MR sequences, 5 out of 60 intact segments showed areas of high signal intensity in the interspinous space communicating with the facet joints (Fig. 4), which previous observers have considered to be markers of PLC inflammatory syndrome (24).

\section{Comparison of Operative and MRI Findings \\ Dissection confirmed that 40 out of 60 ISL segments}




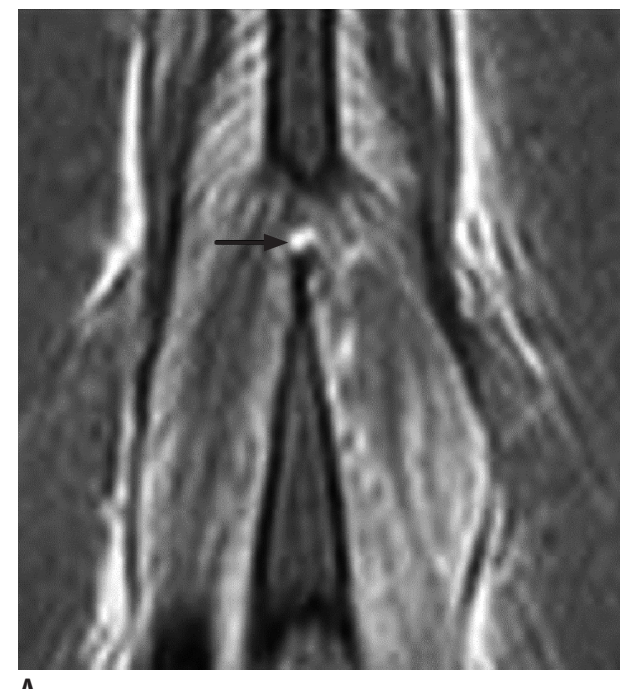

A

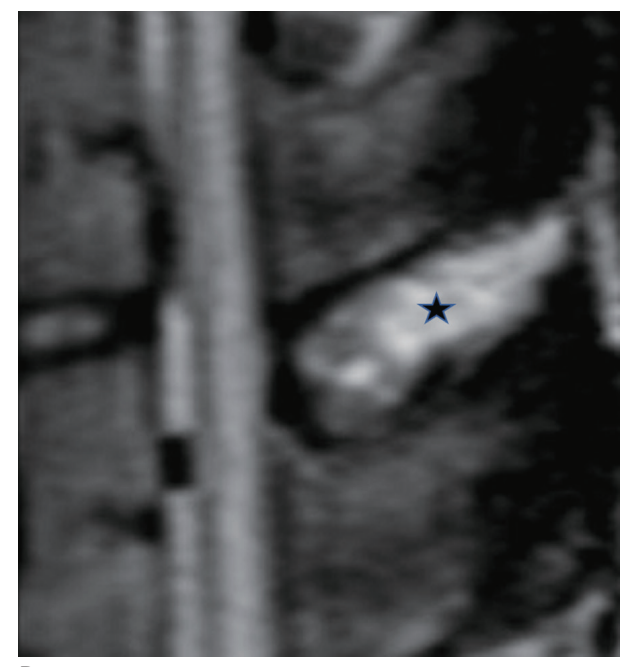

D

Fig. 2. ISL injuries displayed using different magnetic resonance techniques.

Coronal 3D-PDW-SPIR (A) and STIR (B) images show discontinuity of black stripe (i.e., ISL) and discrete fluid crossing ISL (black arrows) indicating ligamentous disruption. On sagittal 3D-PDW-SPIR (C, D) and STIR (E, F) images, area of linear high signal intensity in interspinous space suggests ISL disruption (white arrows), whereas areas of diffuse high signal intensity suggest adjacent soft tissue contusion (stars). STIR = short inversion time inversion recovery, 3D-PDW-SPIR = three-dimensional proton density-weighted spectrally selective inversion recovery

were successfully harvested. In the remaining 20 intact ISL segments, the soft tissues adjacent to the ISL were cut.

Table 1 presents the sensitivity, specificity, and accuracy for ISL injuries diagnosed with different MR techniques. The mean percentages of the sensitivity, specificity, and diagnostic accuracy were $45.0 \%, 70.7 \%$, and $62.1 \%$ for sagittal STIR, $57.5 \%, 91.3 \%$, and $80.0 \%$ for coronal STIR, $100 \%, 96.9 \%$, and $97.9 \%$ for coronal 3D-PDW-SPIR, and $100 \%, 76.3 \%$, and $84.2 \%$ for sagittal 3D-PDW-SPIR (Table 1). Statistically, the sensitivity, specificity, and diagnostic accuracy of the coronal 3D-PDW-SPIR technique for diagnosing ISL tears were significantly greater than those of the sagittal STIR $(p=0.000)$, coronal STIR $(p=0.000)$, and sagittal 3D-PDW-SPIR $(p=0.001)$ techniques (Table 2).

\section{Interobserver Reliability}

The interobserver reliability was substantial to excellent (Table 3).

\section{DISCUSSION}

The PLC is important for maintaining the stability of the thoracolumbar spine. Disruption of the PLC may cause spinal instability, which could potentially require surgery. Although the biomechanical importance of the PLC is not in doubt, controversy still exists regarding the definition 


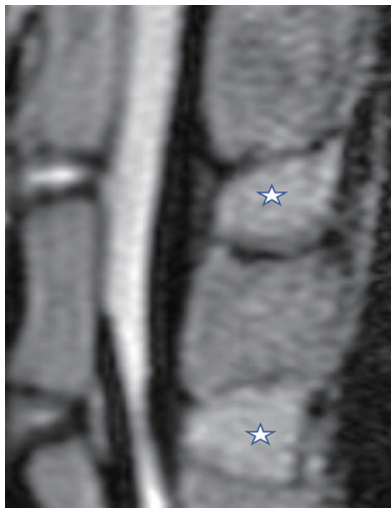

A

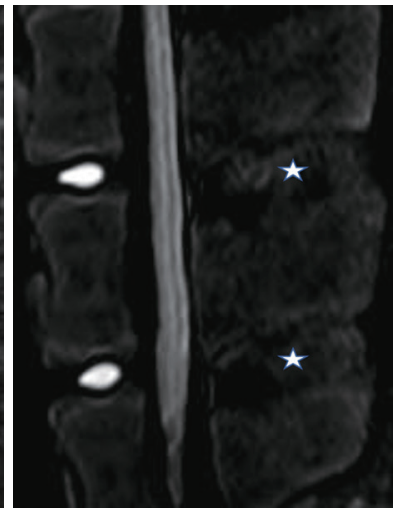

B
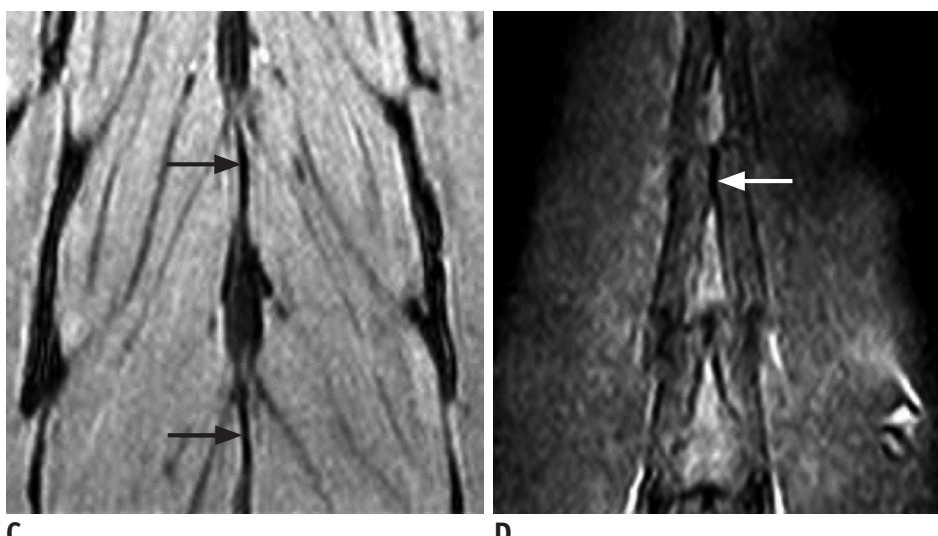

D

Fig. 3. Normal ISL appearance.

Sagittal 3D-PDW-SPIR (A) and STIR (B) images display ISL as diffuse homogeneous low signal intensity (stars) with ill-defined border in interspinous space. On coronal 3D-PDW-SPIR (C) and STIR (D), ISL presents as black stripe (arrows) between spinous processes with well-defined border.

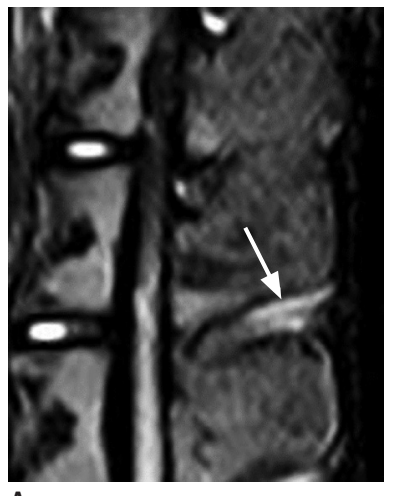

A

Fig. 4. Free fluid in facet joints in normal spinal specimen. Midline sagittal STIR image (A) depicts fluid (white arrow) within ISL communicating with facet joints (black arrows) shown on coronal 3D-PDW-SPIR image (B).

of incompetent PLC as well as the degree of PLC structure damage needed for the diagnosis of PLC disruption. In a previous biomechanical study, spinal instability was created when the ISL and SSL were injured (3). However, previous biomechanical and MRI studies reported that the SSL or LF were the critical PLC components needed to maintain the stability of the thoracolumbar spine $(16,25,26)$. Further biomechanical investigations are needed to resolve these controversies.

Clinics today face the challenge of noninvasively and accurately examining each component of the PLC before surgery. Due to its ability to directly reveal soft tissue injuries, MRI is an effective technique for assessing PLC injuries that cannot be directly detected on plain radiography or CT. In most studies that have used MRI as a diagnostic tool, the SSL and LF were categorized as intact or ruptured; however, the ISL and FC were divided into three categories, including intact, indeterminate, or ruptured $(2$, $5,8,9,12,16-23)$. The "indeterminate" category included structures that were neither clearly intact nor ruptured. Indeterminate PLC status (i.e., totaling four points on the TLICS) represents a common dilemma facing practitioners who manage patients with thoracolumbar burst fractures without neurological damage. Moreover, the indeterminate pattern is likely to create discrepancies between studies. For diagnostic accuracy analysis, two categories are needed. To achieve this, the authors may include the indeterminate group with the ruptured group, which results in measurement bias (9). These issues highlight the need for further development of consensus-based criteria to define MRI features of PLC disruption or integrity (15).

Almost all previous studies evaluated PLC integrity based on sagittal T1-weighted, T2-weighted, and STIR images as well as axial T2-weighted images (2, 8, 9, 12, 16-23), which are routine clinical spinal sequences. A few studies also included coronal T2-weighted images; however, the diagnosis still relied on sagittal images $(9,16)$. On sagittal T1-weighted, T2-weighted, or STIR imaging, the SSL and LF appeared as black stripes. Therefore, a ligamentous tear appeared as a clear disruption of the black stripe or discrete fluid crossing the location of the ligament on fluid-sensitive sequences. However, the ISL cannot be clearly demonstrated in the sagittal plane because it is lamellar in shape and sagittally oriented (27). In cases with paraspinal edema or hemorrhage, sagittal STIR always shows hyperintensity in the interspinous space whether the ISL is disrupted or not due to the partial volume effect. For this reason, ISL integrity has been categorized as indeterminate in some patients. 
Table 1. Diagnostic Performance in Detection of ISL Injuries According to MR Parameters*

\begin{tabular}{lccc}
\hline & Sensitivity & Specificity & Accuracy \\
\hline $\begin{array}{l}\text { Sagittal STIR } \\
\text { Reader A }\end{array}$ & $42.5(17 / 40)$ & & $60.8(73 / 120)$ \\
$\quad$ Reader B & $47.5(19 / 40)$ & $71.3(57 / 80)$ & $63.3(76 / 120)$ \\
$\quad$ Mean & 45.0 & 70.7 & 62.1 \\
Coronal STIR & & & \\
$\quad$ Reader A & $55.0(22 / 40)$ & $90.0(72 / 80)$ & $78.3(94 / 120)$ \\
$\quad$ Reader B & $60.0(24 / 40)$ & $92.5(74 / 80)$ & $81.7(98 / 120)$ \\
$\quad$ Mean & 57.5 & 91.3 & 80.0 \\
Coronal 3D-PDW-SPIR & $100(40 / 40)$ & $96.3(77 / 80)$ & $97.5(117 / 120)$ \\
$\quad$ Reader A & $100(40 / 40)$ & $97.5(78 / 80)$ & $98.3(118 / 120)$ \\
$\quad$ Reader B & 100 & 96.9 & 97.9 \\
\hline Mean & $100(40 / 40)$ & $75.0(60 / 80)$ & $83.3(100 / 120)$ \\
Sagittal 3D-PDW-SPIR & $100(40 / 40)$ & $77.5(62 / 80)$ & $85.0(102 / 120)$ \\
$\quad$ Reader A & 100 & 76.3 & 84.2 \\
\hline Reader B & & & \\
\hline Mean & & & \\
\hline
\end{tabular}

*Main values are presented as percentages. ISL = interspinous ligament, MR = magnetic resonance, STIR = short inversion time inversion recovery, 3D-PDW-SPIR = three-dimensional proton density-weighted spectrally selective inversion recovery

Table 2. Comparison of Diagnostic Performance in Diagnosing ISL Tears among Different MRI Techniques

\begin{tabular}{|c|c|c|c|c|}
\hline & & & & \\
\hline & $\chi^{2}$ & $P^{*}$ & $\chi^{2}$ & $P^{*}$ \\
\hline All four groups & 71.16 & $<0.001$ & 109.77 & $<0.001$ \\
\hline Sagittal 2D vs. coronal 2D & 11.25 & 0.010 & 11.55 & 0.009 \\
\hline Sagittal 2D vs. coronal 3D & 51.93 & $<0.001$ & 49.38 & $<0.001$ \\
\hline Sagittal 2D vs. sagittal 3D & 32.78 & $<0.001$ & 29.29 & $<0.001$ \\
\hline Coronal 2D vs. coronal 3D & 25.67 & $<0.001$ & 68.95 & $<0.001$ \\
\hline Coronal 2D vs. sagittal 3D & 29.46 & $<0.001$ & 36.46 & $<0.001$ \\
\hline Coronal 3D vs. sagittal 3D & 14.68 & 0.001 & 14.63 & 0.001 \\
\hline
\end{tabular}

* Significance level was 0.05 for comparisons among four groups and it was adjusted to 0.008 for all pairwise comparisons in order to control overall family error rate. $2 \mathrm{D}=$ two-dimensional, $3 \mathrm{D}=$ three-dimensional

Table 3. Interobserver Reliability by MRI Technique

\begin{tabular}{lcc}
\hline \multicolumn{1}{c}{ Imaging Technique } & Kappa Value* $^{*}$ & $P$ \\
\hline Sagittal STIR & $0.776(0.651-0.901)$ & $<0.001$ \\
Sagittal 3D-PDW-SPIR & $0.900(0.822-0.978)$ & $<0.001$ \\
Coronal STIR & $0.852(0.746-0.958)$ & $<0.001$ \\
Coronal 3D-PDW-SPIR & $0.949(0.892-1.000)$ & $<0.001$ \\
\hline
\end{tabular}

${ }^{*}$ The $95 \%$ confidence interval is given in parentheses.

In our study, the ISL appeared as a thin black stripe with a well-defined border on coronal STIR and 3D-PDW-SPIR images. Thus, the existence of a black stripe disruption or discrete fluid crossing the midline can serve as a strong basis for an ISL tear. The present study showed that in cases in which only the soft tissue adjacent to the ISL was cut, coronal images were more specific than sagittal images in distinguishing this tissue from an ISL tear. The coronal
3D-PDW-SPIR technique had significantly higher diagnostic specificity and accuracy than the sagittal 3D-PDW-SPIR technique. The coronal STIR was superior to the sagittal STIR; however, the difference was not significant.

Considering the small size of the ISL, the 3D MR images with thin slice thicknesses and no slice gaps were superior to the $2 \mathrm{D}$ images for ligament visualization. In the present study, one 2D image was $3.5 \mathrm{~mm}$ thick with a $3.5-\mathrm{mm}$ slice gap, which corresponded to 4-5 contiguous 3D images with an isotropic slice thickness of $1.6 \mathrm{~mm}$. Therefore, a tear of the ISL could be demonstrated in multiple slices on 3D imaging, whereas it may only be seen in a single slice on $2 \mathrm{D}$ imaging. Our investigation revealed that coronal and sagittal 3D images had significantly better diagnostic sensitivity, specificity, and accuracy than those of coronal and sagittal 2D images. 
In this study, neither 2D nor 3D MR images clearly depicted the FC due to its size. It is possible that 3D images acquired with a 3T MRI scanner could provide more FC details. In most previous studies, articular fluid was considered to be an indication of FC injury $(2,8,9,16$, 17). However, liquid in the facet joint is very common in many patients without trauma and, as shown in our investigation, even exists in the goat spine. This finding may be associated with PLC inflammatory syndrome (24). Since direct signs of FC disruption are very hard to observe, its diagnosis might still have to be based on indirect signs, such as facet joint diastasis or joint space increases (19).

This study had several limitations. First, a goat thoracolumbar spine was used. As shown by MR images, the goat ISL is thinner and has a higher fat content than the human ISL. Theoretically, thicker ligaments would be easier to visualize. Moreover, we performed an animal study instead of a clinical investigation because suspected ligamentous disruption diagnosed by MRI could not be confirmed in patients without surgery. Second, in this study, ISL disruption was created by percutaneous resection instead of a free-falling impact. Although the resection method does not specifically imitate a clinical injury, it has good repeatability. Moreover, the use of a free-falling method may cause vertebral fractures or increase the interspinous space, which could potentially produce bias.

In conclusion, the coronal 3D-PDW-SPIR technique had significantly greater diagnostic accuracy for ISL disruption compared to the sagittal 3D-PDW-SPIR and STIR techniques as well as the coronal STIR technique. In clinical settings, the addition of coronal 3D-PDW-SPIR to a routine MR protocol may be critical for identifying PLC disruption in cases of extensive nearby contusion.

\section{Conflicts of Interest}

The authors have no potential conflicts of interest to disclose.

\section{ORCID iDs \\ Jichen Wang \\ https://orcid.org/0000-0001-5370-056X \\ Xuee Zhu \\ https://orcid.org/0000-0003-2060-9078}

\section{REFERENCES}

1. Whang PG, Vaccaro AR, Poelstra KA, Patel AA, Anderson
DG, Albert TJ, et al. The influence of fracture mechanism and morphology on the reliability and validity of two novel thoracolumbar injury classification systems. Spine (Phila Pa 1976) 2007;32:791-795

2. Rihn JA, Yang N, Fisher C, Saravanja D, Smith H, Morrison WB, et al. Using magnetic resonance imaging to accurately assess injury to the posterior ligamentous complex of the spine: a prospective comparison of the surgeon and radiologist. $\mathrm{J}$ Neurosurg Spine 2010;12:391-396

3. James KS, Wenger KH, Schlegel JD, Dunn HK. Biomechanical evaluation of the stability of thoracolumbar burst fractures. Spine (Phila Pa 1976) 1994;19:1731-1740

4. Oner FC, van Gils AP, Faber JA, Dhert WJ, Verbout AJ. Some complications of common treatment schemes of thoracolumbar spine fractures can be predicted with magnetic resonance imaging: prospective study of 53 patients with 71 fractures. Spine (Phila Pa 1976) 2002;27:629-636

5. Vaccaro AR, Lehman RA Jr, Hurlbert RJ, Anderson PA, Harris $M$, Hedlund $R$, et al. A new classification of thoracolumbar injuries: the importance of injury morphology, the integrity of the posterior ligamentous complex, and neurologic status. Spine (Phila Pa 1976) 2005;30:2325-2333

6. Savage JW, Moore TA, Arnold PM, Thakur N, Hsu WK, Patel AA, et al. The reliability and validity of the thoracolumbar injury classification system in pediatric spine trauma. Spine 2015; 40:E1014-E1018

7. Lee HM, Kim HS, Kim DJ, Suk KS, Park J0, Kim NH. Reliability of magnetic resonance imaging in detecting posterior ligament complex injury in thoracolumbar spinal fractures. Spine (Phila Pa 1976) 2000;25:2079-2084

8. Pizones J, Zúñiga L, Sánchez-Mariscal F, Alvarez P, Gómez-Rice A, Izquierdo E. MRI study of post-traumatic incompetence of posterior ligamentous complex: importance of the supraspinous ligament. Prospective study of 74 traumatic fractures. Eur Spine J 2012;21:2222-2231

9. Pizones J, Sánchez-Mariscal F, Zúñiga L, Álvarez P, Izquierdo E. Prospective analysis of magnetic resonance imaging accuracy in diagnosing traumatic injuries of the posterior ligamentous complex of the thoracolumbar spine. Spine (Phila Pa 1976) 2013;38:745-751

10. Dai LY, Ding WG, Wang XY, Jiang LS, Jiang SD, Xu HZ. Assessment of ligamentous injury in patients with thoracolumbar burst fractures using MRI. J Trauma 2009;66:1610-1615

11. Lee JY, Vaccaro AR, Schweitzer KM Jr, Lim MR, Baron $E M$, Rampersaud $R$, et al. Assessment of injury to the thoracolumbar posterior ligamentous complex in the setting of normal-appearing plain radiography. Spine J 2007;7:422427

12. Haba H, Taneichi H, Kotani Y, Terae S, Abe S, Yoshikawa H, et al. Diagnostic accuracy of magnetic resonance imaging for detecting posterior ligamentous complex injury associated with thoracic and lumbar fractures. J Neurosurg 2003;99(1 Suppl):20-26 
13. Crosby CG, Even JL, Song Y, Block JJ, Devin CJ. Diagnostic abilities of magnetic resonance imaging in traumatic injury to the posterior ligamentous complex: the effect of years in training. Spine J 2011;11:747-753

14. Van Middendorp JJ, Patel AA, Schuetz M, Joaquim AF. The precision, accuracy and validity of detecting posterior ligamentous complex injuries of the thoracic and lumbar spine: a critical appraisal of the literature. Eur Spine J 2013;22:461-474

15. Vaccaro AR, Rihn JA, Saravanja D, Anderson DG, Hilibrand AS, Albert TJ, et al. Injury of the posterior ligamentous complex of the thoracolumbar spine: a prospective evaluation of the diagnostic accuracy of magnetic resonance imaging. Spine (Phila Pa 1976) 2009;34:E841-E847

16. Pizones J, Izquierdo E, Sánchez-Mariscal F, Zúñiga L, Álvarez P, Gómez-Rice A. Sequential damage assessment of the different components of the posterior ligamentous complex after magnetic resonance imaging interpretation: prospective study 74 traumatic fractures. Spine (Phila Pa 1976) 2012;37:E662-E667

17. Rihn JA, Fisher C, Harrop J, Morrison W, Yang N, Vaccaro AR. Assessment of the posterior ligamentous complex following acute cervical spine trauma. J Bone Joint Surg Am 2010;92:583-589

18. Schweitzer KM, Vaccaro AR, Harrop JS, Hurlbert J, Carrino $J A$, Rechtine GR, et al. Interrater reliability of identifying indicators of posterior ligamentous complex disruption when plain films are indeterminate in thoracolumbar injuries. $J$ Orthop Sci 2007;12:437-442

19. Vaccaro AR, Lee JY, Schweitzer KM Jr, Lim MR, Baron EM, Oner $\mathrm{FC}$, et al. Assessment of injury to the posterior ligamentous complex in thoracolumbar spine trauma. Spine J 2006;6:524528
20. Kim NR, Hong SH, Choi JY, Chang BS, Lee JW, Myung JS, et al. Spreading epidural hematoma and deep subcutaneous edema: indirect MRI signs of posterior ligamentous complex injury in thoracolumbar burst fractures. Skeletal Radiol 2010;39:767772

21. Mi J, Sun XJ, Zhang K, Zhao CQ, Zhao J. Prediction of MRI findings including disc injury and posterior ligamentous complex injury in neurologically intact thoracolumbar burst fractures by the parameters of vertebral body damage on CT scan. Injury 2018;49:272-278

22. Rosenthal BD, Boody BS, Jenkins TJ, Hsu WK, Patel AA, Savage JW. Thoracolumbar burst fractures. Clin Spine Surg 2018;31:143-151

23. Dawkins RL, Miller JH, Ramadan OI, Lysek MC, Kuhn EN, Rocque $B G$, et al. Thoracolumbar injury classification and severity score in children: a reliability study. $J$ Neurosurg Pediatr 2018;21:284-291

24. Lehman VT, Murthy NS, Diehn FE, Verdoorn JT, Maus TP. The posterior ligamentous complex inflammatory syndrome: spread of fluid and inflammation in the retrodural space of Okada. Clin Radiol 2015;70:528-535

25. Li Y, Shen Z, Huang M, Wang X. Stepwise resection of the posterior ligamentous complex for stability of a thoracolumbar compression fracture: an in vitro biomechanical investigation. Medicine (Baltimore) 2017;96:e7873

26. Wu CC, Jin HM, Yan YZ, Chen J, Wang K, Wang JL, et al. Biomechanical role of the thoracolumbar ligaments of the posterior ligamentous complex: a finite element study. World Neurosurg 2018;112:e125-e133

27. Scapinelli R, Stecco C, Pozzuoli A, Porzionato A, Macchi V, De Caro R. The lumbar interspinous ligaments in humans: anatomical study and review of the literature. Cells Tissues Organs 2006;183:1-11 